\title{
Responses to Higher Concentration Lecithin of Apoptosis Relevant Factors and Detection of Specific Lecithin Binding Proteins by AC-MS assay
}

\author{
Yuxi $\mathrm{Yu}^{1, *}$ \\ ${ }^{1}$ College of Life Sciences, South China Agricultural University, Guangzhou, 510614, China
}

\begin{abstract}
Lecithin in mixed micelles attenuates cytotoxicity by interacting with proteins or other cell inclusions. The reaction was then captured via magnetic beads binding approach. This research proposal was designed to discover the impacts of lecithin on apoptosis relevant factors (including caspase 3/8/9 and PARP) by Western Blot and specific protein binding mechanism of lecithin detoxification pathway, using caco-2 cell model. Preliminary investigation of the properties of the proteins was conducted by MS (Mass Spectrometry) couplet to AC (Affinity Chromatography) assay. Further research of particular proteins was to identify the size and peptide sequence. This study also provided solid evidence for the previous research result, which was lecithin reduce cytotoxicity induced by SDC contained mixed micelles.
\end{abstract}

\section{Introduction}

It is believed that if Lecithin exists in mixed micelles, then it has the ability to attenuate the cytotoxicity of bile salts that is one of the ingredients in the mixed micelles, which can be widely used as important drug delivery systems by enhancing the permeability and solubility of some insoluble oral drugs across gastrointestinal epithelia[1][2].

The previous cell model that has been used are Caco2 cells and could be further applied to human gastrointestinal epidermal cells[6]. Therefore, SDC (Sodium Deoxycholate), also known as bile salts, has cytotoxicity when utilized as an essential role in drug carriers, while Lecithin in contrast can reduce a certain degree of this toxicity.

So as far as it is to be known, Lecithin is not a bad chemical compound for human body after all, while the premise is at an appropriate moderate dose [8]. Furthermore, the molecular mechanism of how Lecithin inhibits the toxic effect of bile salts is also a valuable motif to be discussed and researched.

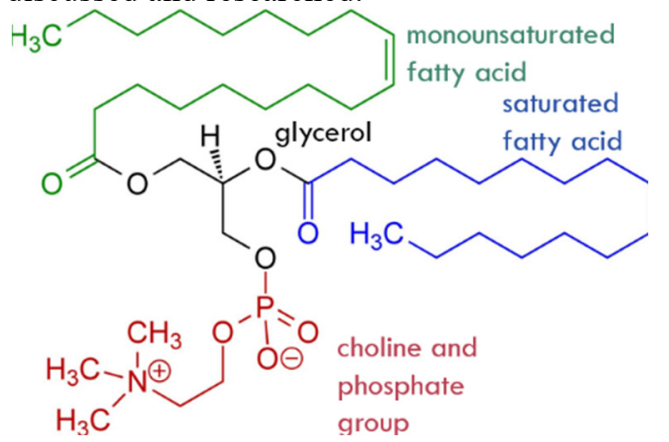

Fig.1. An example of a phosphatidylcholine, a type of phospholipid in lecithin
Different concentrations of mixed micelles culture medium without fetal bovine serum were prepared by mixing different ratio of different concentrations of Lecithin and SDC. Also, Caco-2 cells were usually cultured in a normal animal cell culture medium and conditions with fine nutrition. And the number of 45-52 passage of cells were taken for such experiment [9].

In conclusion, to further summarize and discuss, with the existence of SDC in mixed micelles, cell viability and proliferation can be inhibited, tight junction can be broken, apoptosis can be accelerated, but lecithin in mixed micelles can attenuate all of those negative impacts. Hence, optimum use of lecithin in the Lecithin/SDC mixed micelles is rather significant to attenuate the cytotoxicity.

However, when it comes to real applications as oral drug carriers, the molecular mechanisms of how Lecithin reducing the cytotoxicity need to be considered and investigated. Therefore, in this study, we designed a series of experiments to evaluate the of impact of lecithin on molecular level and experimental verifications of what Lecithin specific binding protein is. And it was integrated with the former cellular level results to better make clear of protein level interaction.

\section{Methods and materials}

\subsection{Chemicals and regents}

Lecithin (LipiodS100), Fetal Bovine Serum (SH30070. 03), Trypsin (RPT-0201), Phosphate Buffer Saline (Shanghai, China), Dimethyl Sulfoxide (USA), Sepharose 4B antibody (Shanghai, China), Dithiothreitol (DITH-703211), Sodium Dodecyl Sulfate (China), Magnetic beads (PuriMag), Caco-2 cells (from human colon cancer cells)

\footnotetext{
*Corresponding Author's E-mail: yxyu@ $@$ scau.edu.stu.cn
} 


\subsection{Caco-2 cells cell culture}

Caco-2 cells originated from human colonic carcinoma was cultured under absolute sterile and non-toxic environment with the treatment of a certain amount of antibiotics. And the physical conditions of culture cells were optimal temperature and $\mathrm{PH}\left(36.5 \pm 0.5^{\circ} \mathrm{C}, 7.2 \sim 7.4\right)$, ( $5 \%$ of $\mathrm{CO}_{2}$ in a humidified atmosphere is to maintain the $\mathrm{pH}$ stability of the culture, and the rest of gas was air). The chemical nutrition for the incubated cells were DMEM (Dulbecco's modified minimum essential medium) with other additional substances, including $10 \%$ fetal bovine serum (FBS), $1.5 \%(\mathrm{v} / \mathrm{v})$ of nonessential amino acids and antibiotics (streptomycin $100 \mathrm{mg} / \mathrm{ml}$ and penicillin $200 \mathrm{mg} / \mathrm{ml}$ ). The culture solution was placed in flasks, where the cells were grown in. To ensure no accumulation of toxic substances and better nutrition, the culture medium was replaced each day. And treatment of cells with trypsin every five days for passage. So, the cells with the passage around 50 would be used in the next experiments.

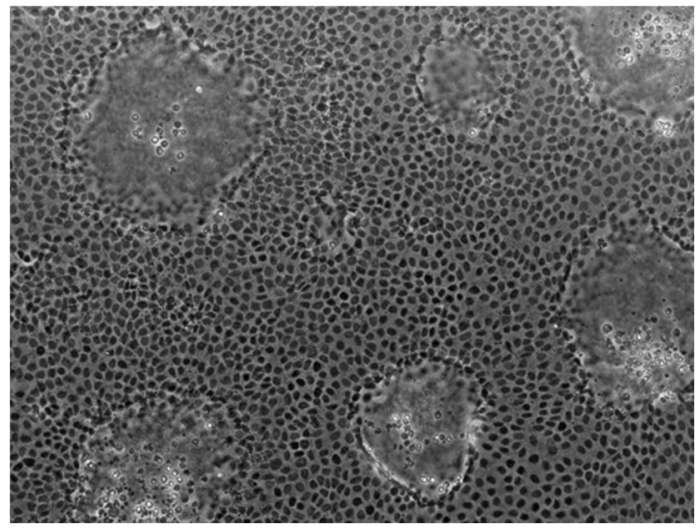

Fig.2. CaCo-2 cells imaged with phase contrast microscopy using 100X magnification

$\mathrm{CaCo}-2$ cells in this image had been kept in culture for 21 days, allowing them to grow confluent and epithelialize. (researchgate.net)

\subsection{Assessment of Caspase 3/8/9}

Caspases are a family of proteases that play an important role in cell death, which is mediated by pyroptosis, necroptosis and autophagy [11]. The apoptosis factors Caspase-3/8/9 was measured with the increasing concentration of Lecithin to demonstrate Lecithin can prevent apoptosis. Caspase- 3 has the function of cell differentiation and tissue regeneration in different ways which have no sign of cell apoptosis. Caspase- 8 on the other way, mediates cell death in both programmed cell death (PCD) and necroptosis. Caspase-9 is an upstream caspase in the process of apoptosis signal transduction [12].

Caco-2 cells (about one $200-\mu \mathrm{L}$ culture flask cell volume) were incubated into well plates. After 3-day of cell growth, the cells were treated with same volume of Lecithin/SDC mixed micelles and physiological saline in replace of culture medium and then 2 hours later, $20 \mu \mathrm{L}$ of distinct concentration of Lecithin was added to every plate.
After another 3-hour of treatment, all medium would be removed and the cells were washed by $150 \mu \mathrm{L}$ of DMSO to dissolve remnants. Then, the cells were collected after centrifugation for 5 minutes, and the supernatant was aspirated and washed with PBS. After the supernatant was taken, $100 \mu \mathrm{L}$ lysate was added to each cell sample to resuspend the precipitation, and the cleavage of cells were processed in ice bath for 15 minutes. And then the extract was centrifuged at $4^{\circ} \mathrm{C} 16,000 \mathrm{~g}$ for 15 minutes, the supernatant was transpired to the pre-cooled centrifuge tube in the ice bath. The enzyme activity of caspase- 3 was determined immediately. At the same time, a small number of samples were taken to measure the protein concentration with the Bradford method.

\subsection{Assessment PARP (poly adp ribose polymerase) by western blot}

The degradation of PARP is one of the markers of apoptosis. It is a zymolyte of Caspase, which can cut PARP into 2 segments, resulting in the fracture of DNA. The cleavage of PARP has been tested in order to prove Lecithin's function of alleviating mixed micelles' cytotoxicity. Caco-2 cells were prepared in the same way as Caspase 3/8/9 test before. The cells without any mixed micelles was a control group. And then after the culture, gradients of different concentration of Lecithin were added into each well plates for 2 hours, and a negative control group is that without any more Lecithin addition. Then cell lysis was thereafter proceeded and underwent Western Blot assay, which firstly was conducted by the specific hybridizing antigen and antibody to label PARP. The test of Western Blot was performed in triplicate for every sample.

\subsection{Detection of lecithin specific binding protein by affinity chromatography(ac) and mass spectrometry(ms)}

The Caco- 2 cells were incubated in well plates, and they have been cultured for. First, the whole proteins of cells should be extracted. A phosphate buffer solution of $0.02 \%$ ethylenediamine tetra acetic acid was used to digest and collect cells. And then directly cell centrifugation was conducted and the cells were washed by cold PBS solution three times before cell counting. The cells then were collected into centrifugal tube for $5 \mathrm{~min}$ centrifugation (1000rmp/min). Enzymolysis of cells was in progress in cell lysate. $(10 \mu \mathrm{L}$ phosphatase inhibitors, $1 \mu \mathrm{L}$ protease inhibitors and $5 \mu \mathrm{L} 100 \mathrm{mM}$ PMSF per millilitre) under ice bath condition. Proteins were extracted right before centrifugation for $15 \mathrm{~min}$ (14000rmp). The isolate was then repacked and stored in $-70^{\circ} \mathrm{C}$ refrigerator.

The method of using affinity chromatography coupled to mass spectrometry identified proteins that interact with Lecithin. The protein mixture was treated with Lecithin foe $3 \mathrm{~h}$ before the experiment begun. $10 \mathrm{ml}$ of protein solution was taken and mixed with $1 \mathrm{ml}$ Sepharose 4B with labeled control antibody, and then placed on vertical levitator at $4^{\circ} \mathrm{C}$ for $2 \mathrm{~h}$. After $1 \mathrm{~min}$ of $2000 \mathrm{rmp} / \mathrm{min}$ centrifugation, each sample was added Sepharose 4B 
labeled with monoclonal antibody and blending overnight. The labeled Sepharose 4B was collected from precipitate after $1 \mathrm{~min}$ of $10000 \mathrm{r} / \mathrm{min}$ centrifugation. It was washed with detergent 3 times, and treated with $1 \mathrm{ml}$ glycine-HCL for $5 \mathrm{~min}$. Then, centrifugal supernatant was extracted (10 min $10000 \mathrm{r} / \mathrm{min}$ centrifugation). At last, $3 \mathrm{ml}$ eluent with monoclonal antibody labeled was obtained, and soon it was concentrated 100 times to $30 \mu \mathrm{L}$. The remaining concentrate was taken out and returned to room temperature and added into affinity chromatography colloid. It underwent SDS-PAGE with DTT existence and the gel was dyed.

Small patches from protein band of SDS-PAGE gel were cut to further analyze enzymatic peptide sequence by using tandem mass spectrometry coupled with electro spray (LS-ESI-MS/MS). And then the size of the protein would be measured.

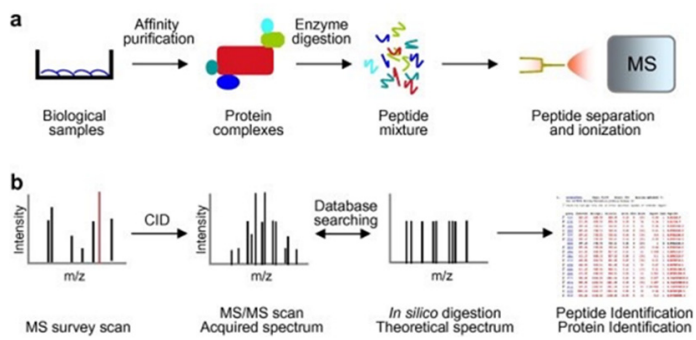

Fig.3. Diagram map of Affinity chromatography coupled with Mass Spectrometry assay (sciencedirect.com)

\subsection{Examination of lecithin beads binding protein}

Lecithin compounds were conjugated to magnetic beads. Caco-2 cells lysates were purified to obtain preliminary proteins, and the complete protein substances were extracted like the previous step. And then pour cell lysates over the beads to distinguish Lecithin sticking protein. After all mixed substances were mixed well, the beads were then drawn away by magnet. The Lecithin/protein/bead mix were washed by PBS to get rid of other impurities and buffer to extract binding protein respectively. Then, using Western Blot assay to analyze the size of the protein was necessary to identify the protein.

\section{Possible results}

\subsection{Effects increasing concentration of lecithin on Caspase $3 / 8 / 9$ and parp cleavage}

In the caspase $3 / 8 / 9$ experiment, a total of eight possible results were summed up. To be specific, all the caspases that have been tested were blocked or loss-of-function, part of the caspases were blocked (one or two of these caspases were suppressed and the rest of caspases were in normal function) and none of the caspase 3/8/9 was blocked.

PARP was either broken or well-functioned, whereas lecithin was supposed to have positive effects on cell growth, so the PARP breakage are not likely to occur apparently.

In caco- 2 cells, the overexpression of caspase and the fracture of PARP are both indications of cell death. So, by verifying lecithin could block caspases and prevent PARP from rupturing, it would be a strong argument to prove the previous hypothesis that lecithin can leads to less apoptosis. It probably turned out higher concentration of lecithin led to less caspase expression and less PARP damage. And the result could also be an implication of lecithin as an ingredient in mixed micelles applications.

\subsection{Result of lecithin specific binding protein}

The experiment utilized AC-MS assay was to find out whether some proteins bound to lecithin or not, and the conclusion is between them. The work of lecithin has shown its clear way that some kind of chemical reaction pathway should be unfold to play such a role of detoxification, which illustrated the proper function of lecithin was an improbability to bind nothing. When it comes to the accurate biochemical reaction and its steps, research outcomes among this fields have not been discovered yet. If more than one protein had the capability to bind lecithin, then lecithin' actions towards these proteins could be numerous possibilities, for instance, difference reactions in vivo and in vitro or in intact cells and cell lysis could be entirely different or similar or just the same.

\subsection{Identification of protein that binds to lecithin-bead complex}

This step of investigation was clearly aimed to further explore the biochemical and physical properties of the particular protein molecule. If none of the proteins coupled to lecithin, then it would be assumed as an evidence that proteins have neutral impact on the whole detoxification reaction, or the actual protein has been degraded or inactivated during the progress. Another theoretical proposal was that only a single protein bound to the lecithin, and that would be critical for the protein and had highly conserved manner, which was believed to be less likely. The third opinion was loads of proteins had the lecithin combining ability, which means more than a single protein can participate in lecithin-attenuatingcytotoxicity mechanism. But the multiple interaction reactions might just occur in vitro, while the number of the proteins that could couple with lecithin could be limited and the mechanism would be much more complex.

\section{Discussion}

It is suggested that lecithin or phosphatidylcholine binds to bile salts both in vivo (in gallbladder) and in vitro (to form mixed micelles drug carriers) can attenuate the bile salts-induced toxicity [5][16]. The way it worked in living tissue is either by enhancing the hydrophobic properties of mucosa or accelerating the formation of less cytotoxic mixed micelles [4]. Also, the mixed micelles are of significance medical value to serve as carriers for insoluble drugs to facilitate the oral drugs' absorption and solubilization. In fact, the increase in drug molecules solubility and permeability across epithelia are mainly 
coupled with mixed micelles' delivery capacity, which results in the increase of drugs' bioavailability $[17][18][19]$. However, explicit studies about the mixed micelles' influences on gastrointestinal epithelia have not been revealed in detail, and the potential and unknow injury to tissues and organs has been lacking to investigate.

In this paper, we laid particular emphasis on the mechanism of Lecithin reducing cytotoxicity as a role in drug deliver mixed micelles. To be more specific, it was aimed to test whether it binds to a protein or not. The teat of Caspase 3/8/9 and PARP cleavage have been done to evaluate apoptosis which was meant to provide evidence for Lecithin' potential function of preventing cell death. By increasing the concentration of Lecithin, caspase 3/8/9 and PARP cleavage have shown evidently a lower expression level, indicating Lecithin can slow cell death. This result is similar as the previous report that more lecithin in mixed micelles can cause less apoptosis [1]. Furthermore, to understand the long-term effects of higher lecithin concentration treatment, the cells have been exposure to lecithin for another 3 more days. The cells in relatively higher concentration of lecithin had a significant slower apoptosis, which is relevant to the initial concentration of SDC as well as mixed micelles and actuation time of subsequent lecithin treatment. The result also implies that cytotoxicity of mixed micelles that induced apoptosis can be relieved by lecithin. Moreover, Caco-2 cells are from human colon cancer tissue, which cannot represent differentiated cells like human gastrointestinal cells. Therefore, cell culture time should be extended until cell differentiation, which could be better simulation of true human in vivo cells [22].

To further prove our hypothesis, another experiment about examining macromolecule by AC-MS has been processed. It is known that mixed micelles injury gastrointestinal tissues by damaging tight junctions, which is an essential barrier that can control the paracellular transport of materials and nutrients by size and electric charge [20][21]. Therefore, the damage can be effectively and specifically inhibited by lecithin-reducingcytotoxicity pathway, which is not yet discovered. But finding the key protein that interact with lecithin compound was a leaped advance. In this study, the different proteins were screened and the specific protein bound to lecithin was identified by Affinity Chromatography and then purified and examined by Mass Spectrometry and Western Blot. Among all the possible results, a good explanation of specific interaction coupled with signaling pathway can hold the most powerful support for our hypothesis.

All protein in the cell were extracted by cell lysis, and it must be certain that cell lysis was without any cleavage and decomposition of protein. Protein extraction is an important step in protein analysis and also an important part of other protein analysis, which is regarded as the breakthrough direction of diseases in many biochemical researches. In this experiment, lecithin was attached to a bead and mixed with cell lysates, and the specific protein was identified.

\section{Conclusion}

Cell apoptosis can be attenuated by lecithin. With the treatment of SDC/Lecithin mixed micelles, SDC can cause the abnormal expression of caspase $3 / 8 / 9$ and the cleavage of PARP, which are both molecular signals of apoptosis, and both of which are expected to be inhibited by adding relatively higher concentration of lecithin. And the specific binding protein or proteins are detected by the combination method of AC and MS and magnetic beads involved biochemical measurement.

\section{References}

1. Ya'nan Tan, Jianping Qi, Yi Lu, Fuqiang $\mathrm{Hu}$, Zongning Yin, Wei Wu. Lecithin in mixed micelles attenuates the cytotoxicity of bile salts in Caco-2 cells[J]. Toxicology in Vitro,2013,27(2).

2. van Hasselt, P.M., Janssens, G.E.P.J., Slot, T.K., van der Ham, M., Minderhoud, T.C., Talelli, M., Akkermans, L.M., Rijcken, C.J.F., van Nostrum, C.F., 2009. The influence of bile acids on the oral bioavailability of vitamin $\mathrm{K}$ encapsulated in polymeric micelles. J. Control. Release 133, 161168.

3. Duane, W.C., 1980. Mechanism by which bile-salts disrupt the gastric-mucosal barrier. Gastroenterology 78, 1159-1159.

4. Dial, E.J., Rooijakkers, S.H.M., Darling, R.L., Romero, J.J., Lichtenberger, L.M., 2008. Role of phosphatidylcholine saturation in preventing bile salt toxicity to gastrointestinal epithelia and membranes. J. Gastroen. Hepatol. 23, 430-436.

5. Narain, P.K., DeMaria, E.J., Heuman, D.M., 1998. Lecithin protects against plasma membrane disruption by bile salts. J. Surg. Res. 78, 131-136.

6. Turco, L., Caloni, F., Consiglio, E.D., Testai, E., Stammati, A., 2010.Caco-2/TC7cellline characterization for intestinal absorption: how reliable is this in vitro model for the prediction of the oral dose fraction absorbed in human? Toxicol. In Vitro.

7. Norum Kaare R. The function of lecithin: cholesterol acyltransferase (LCAT).[J]. Scandinavian journal of clinical and laboratory investigation,2017,77(4).

8. Majdolhosseini Leila, Ghasemi Hossein Ali, Hajkhodadadi Iman, Moradi Mohammad Hossein. Nutritional and physiological responses of broiler chickens to dietary supplementation with de-oiled

9. Liu Shangyi, Liu Xiuwen, Cai Yun, Song Haifeng, Ying Wantao, Zhu Baozhen, Li Lei, Tang Zhongming, Qian Xiaohong. Extraction and Characterization of Rh-Endostatin in Rhesus Serum by Affinity Chromatography Combined with Peptide Mass Fingerprinting. Chinese Journal of Analytical Chemistry, 2003, 31(3): 277-282.

10. Shah, P., Jogani, V., Bagchi, T., Misra, A., 2006. Role of Caco-2 cell monolayers in prediction of intestinal drug absorption. Biotechnol. Prog. 22, 186-198. 
11. Mehrzad Jalil, Fazel Fatemeh, Pouyamehr Nazaninzeynam, Hosseinkhani Saman, Dehghani Hesam. Naturally Occurring Level of Aflatoxin B1 Injures Human, Canine and Bovine Leukocytes Through ATP Depletion and Caspase Activation.[J]. International journal of toxicology,2020,39(1).

12. Nehal M. Elsherbiny, Mohammed M.H. Al-Gayyar, Khaled H. Abd El Galil. Nephroprotective role of dipyridamole in diabetic nephropathy: Effect on inflammation and apoptosis[J]. Life Sciences,2015,143.

13. Lippold Steffen, Nicolardi Simone, Wuhrer Manfred, Falck David. Proteoform-Resolved FcrRIIIa Binding Assay for Fab Glycosylated Monoclonal Antibodies Achieved by Affinity Chromatography Mass Spectrometry of Fc Moieties.[J]. Frontiers in chemistry,2019,7.

14. Cestari Igor. Identification of Inositol Phosphate or Phosphoinositide Interacting Proteins by Affinity Chromatography Coupled to Western Blot or Mass Spectrometry.[J]. Journal of visualized experiments: JoVE,2019(149).

15. Xuxia Zhou, Honghan Lin, Shichen Zhu, Xia Xu, Fei Lyu, Yuting Ding. Textural, rheological and chemical properties of surimi nutritionally-enhanced with lecithin[J]. LWT,2019.

16. Barrios, J.M., Lichtenberger, L.M., 2000. Role of biliary phosphatidylcholine in bile acid protection and NSAID injury of the ileal mucosa in rats. Gastroenterology 118, 1179-1186.

17. Pithavala, Y.K., Odishaw, J.L., Han, S.M., Wiedmann, T.S., Zimmerman, C.L., 1995. Retinoid absorption from simple and mixed micelles in the rat intestine. J. Pharm. Sci. 84, 1360-1365.

18. Wiedmann, T.S., Liang, W., Kamel, L., 2002. Solubilization of drugs by physiological mixtures of bile salts. Pharm. Res. 19, 1203-1208.

19. Yu, J.N., Zhu, Y.A., Wang, L., Peng, M., Tong, S.S., Cao, X., Qiu, H., Xu, X.M., 2010. Enhancement of oral bioavailability of the poorly water-soluble drug silybin by sodium cholate/phospholipid-mixed micelles. Acta Pharmacol. Sin. 31, 759- 764.

20. Misra, A., Shah, P., Jogani, V., Bagchi, T., 2006. Role of Caco-2 cell monolayers in prediction of intestinal drug absorption. Biotechnol. Progr. 22, 186-198.

21. Gonzalez-Mariscal, L., Tapia, R., Chamorro, D., 2008. Crosstalk of tight junction components with signaling pathways. BBA-Biomembranes 1778, 729-756.

22. Zolbin Masoumeh Majidi, Ersoy Gulcin Sahin, Aliakbari Fereshte, Amidi Fardin, Daghigh Faezeh, Abbasi Mehdi, Johnson Joshua. Basal characterization and in vitro differentiation of putative stem cells derived from the adult mouse ovary[J]. In Vitro Cellular \& Developmental Biology,2020,56(1). 